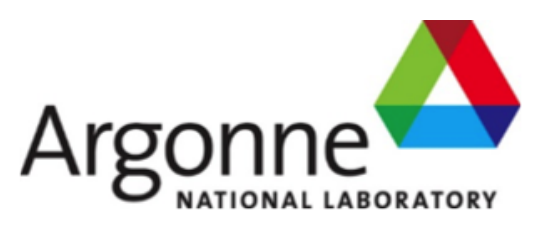

\title{
An Initial Assessment of the Design Margins of Different ASME Section III, Division 5 Design Rules
}

Applied Materials Division 
About Argonne National Laboratory

Argonne is a U.S. Department of Energy laboratory managed by UChicago Argonne, LLC under contract DE-AC02-06CH11357. The Laboratory's main facility is outside Chicago, at 9700 South Cass Avenue, Argonne, Illinois 60439. For information about Argonne and its pioneering science and technology programs, see www.anl.gov.

\section{DOCUMENT AVAILABILITY}

Online Access: U.S. Department of Energy (DOE) reports produced after 1991 and a growing number of pre-1991 documents are available free at OSTI.GOV (http://www.osti.gov/), a service of the U.S. Dept. of Energy's Office of Scientific and Technical Information

Reports not in digital format may be purchased by the public from the National Technical Information Service (NTIS):

U.S. Department of Commerce

National Technical Information Service

5301 Shawnee Rd

Alexandria, VA 22312

www.ntis.gov

Phone: (800) 553-NTIS (6847) or (703) 605-6000

Fax: (703) 605-6900

Email: orders@ntis.gov

Reports not in digital format are available to DOE and DOE contractors from the Office of Scientific and Technical Information (OSTI)

U.S. Department of Energy

Office of Scientific and Technical Information

P.O. Box 62

Oak Ridge, TN 37831-0062

www.osti.gov

Phone: (865) 576-8401

Fax: (865) 576-5728

Email: reports@osti.gov

\section{Disclaimer}

This report was prepared as an account of work sponsored by an agency of the United States Government. Neither the United States Government nor any agency thereof, nor UChicago Argonne, LLC, nor any of their employees or officers, makes any warranty, express or implied, or assumes any legal liability or responsibility for the accuracy, completeness, or usefulness of any information, apparatus, product, or process disclosed, or represents that its use would not infringe privately owned rights. Reference herein to any specific commercial product, process, or service by trade name, trademark, manufacturer, or otherwise, does not necessarily constitute or imply its endorsement, recommendation, or favoring by the United States Government or any agency thereof. The views and opinions of document authors expressed herein do not necessarily state or reflect those of the United States Government or any agency thereof, Argonne National Laboratory, or UChicago Argonne, LLC. 


\section{An Initial Assessment of the Design Margins of Different ASME Section III, Division 5 Design Rules}

Applied Materials Division

Argonne National Laboratory

September 2020

Prepared by

M. C. Messner, Argonne National Laboratory

T.-L. Sham, Argonne National Laboratory 



\section{Abstract}

This report develops a method for assessing the design margin of the ASME Section III, Division 5 Class A design rules, focusing on a definition of margin as the ratio between the actual, expected component life and the ASME design life. Full inelastic finite element simulations with a complete damage model capturing the available creep, creep-fatigue, fatigue, and tension test failure data produce the expected component lives, which can then be compared to corresponding ASME design calculations. The focus of this particular work is on Alloy 617 and the ASME creep-fatigue design rules, but the approach is general and could be applied to other design limits and to other materials. A margin assessment of a representative Alloy 617 component shows the ASME creep-fatigue design rules applied to this component have a margin of 10 on design life. The report describes several challenges in completing a more comprehensive margin assessment of the ASME rules, in particular challenges in developing probabilistic assessment methods for high temperature structural components. The report details these challenge and discusses the immediate possibilities of the deterministic margin assessment method developed here. 

An Initial Assessment of the Design Margins of Different ASME Section III, Division 5 Design Rules September 2020

\section{Table of Contents}

Abstract i i

Table of Contents $\quad$ iii

List of Figures $\quad$ V

List of Tables vii

1 Introduction 1

2 A damage model for Alloy 617

2.1 Damage model derivation . . . . . . . . . . . . . . . 5

2.2 Calibrating the damage model . . . . . . . . . . . . . . . . 7

2.3 Complete inelastic damage model . . . . . . . . . . . . . . . . . . 11

2.4 Evaluating the damage model . . . . . . . . . . . . . . . . 11

3 Example margin determination 15

3.1 The sample problem . . . . . . . . . . . . . . . . . . . . 15

3.2 ASME design by elastic analysis . . . . . . . . . . . . . . . . . 17

3.3 Inelastic damage modeling . . . . . . . . . . . . . . . . . . . . . . . . . . . . . . . . . . . . . . . . . .

3.4 Margin assessment . . . . . . . . . . . . . . . . . 20

4 Conclusions and future work 23

Acknowledgments $\quad 25$

$\begin{array}{ll}\text { Bibliography } & 27\end{array}$ 



\section{List of Figures}

2.1 Example simulation demonstrating the effect of increasing the parameter $n$.

2.2 Initial damage model calibration to the full INL experimental data set. The figure shows points for the work-to-failure and average experimental work rate for all the tests. The tests corresponding to the circled symbols were used to fit the semilog trilinear initial correlation for $W_{\text {crit }}(\dot{W})$. The omitted tests are the cyclic experiments with holds (i.e. creep-fatigue tests). The data shows a moderate temperature effect, which is neglected for the current work.

2.3 Composite model combining the fit to the full INL experimental data and the power law model for the lower-shelf ductility fit to the Larson-Miller correlation. . . . . . . . . . . . . . . . . . . .

2.4 Comparison between the inelastic damage model and the available INL tensile curves at $950^{\circ} \mathrm{C}$. Model is the solid black line, experiments are the dashed lines. . . . . . . . . . . . . . . . . . .

2.5 Comparison between the available creep curves and the model predictions at $950^{\circ} \mathrm{C}$. The solid lines give the model predictions for the corresponding stress levels. The dashed curves are the experimental creep curves. The dotted vertical lines show the rupture life predicted by the Larson-Miller correlation.

2.6 Comparison between the predicted and actual accumulated damage for all the experimental data, including the creep-fatigue tests. . . . . . . . . . .

3.1 Sample problem used for the margin assessment. Figure shows a notional cross-section of a coaxial double tube duct along with the simplified structural model used in the margin assessment. . . . . . . . . . . . . . .

3.2 Temperatures at three locations across the inner tube, plotted as a function of time during the transient. . . . . . . . . . . . . . . . . 17

3.3 Elastically-calculated stresses in the inner tube. . . . . . . . . . . . 18

3.4 Stresses from the full damage calculation over the first repetition of the loading transient. . . . . . . . . . . . . . . . . . . 19

3.5 Extrapolated damage at failure plotted through the tube thickness. . . . . . 20 

An Initial Assessment of the Design Margins of Different ASME Section III, Division 5 Design Rules September 2020

\section{List of Tables}

3.1 Key parameters for the sample problem. . . . . . . . . . . . . . 



\section{Introduction}

This report develops a process for assessing the design margin in the ASME Section III, Division 5, Subsection HB, Subpart B rules covering the design and construction of Class A high temperature nuclear reactor structural components. Design margin, at least for timedependent failure modes, can be expressed as the difference between the actual component time to failure and the component design life. This report characterizes the actual time to failure as a best-estimate prediction based on average material properties. This margin is necessary to cover the uncertainties in the engineering design process, including uncertainties in the actual material properties, the thermal and structural loads during plant operation, and the actual, as-built component geometry. Components with higher safety consequences typically require a larger design margin than those with lower consequences of failure. Design methods for nuclear components, for example, often include a larger design margin than methods for non-nuclear components.

The design margin inherent in the ASME Section III, Division 5 rules for metallic components is largely unknown. The design methods are thought to be generally very conservative, but to our knowledge there has been no work to explicitly quantify the method's design margin. Quantifying the design margin in the rules would provide the necessary initial development to support a Risk Informed, Performance Based (RIPB) licensing approach and a means to better tune the design method. For example, a margin assessment could be used to determine if the design margin remained constant for different temperature ranges, materials, and component types. Ideally, the method would produce a roughly uniform margin when considering different materials and component geometries and the rules could be tuned to produce this result. Moreover, explicitly quantifying the design margin could allow for optimizing the rules to produce more efficient, while still adequate, designs.

This report focuses on the design rules for Class A high temperature components found in Section III, Division 5, Subsection HB, Subpart B. These rules apply to safety significant components and form the basis of other design rules in Division 5 (for example, the rules for Class SM core support structures). Design margin could be quantified in several ways. Classical design methods based on allowable stresses often express the margin in terms of a safety factor on the applied load - for example, the component could withstand a pressure twice as much as the actual design pressure. Alternatively, margin could be expressed in terms of the component geometry - for example, the component is twice as thick as it needs to be. Neither of these approach suits the Class A design rules because: (1) the Division 5 rules work with multiple load cases and time-dependent transient loads; applying a load factor is not straightforward and (2) quantifying design margin in terms of the component geometry only works for simple components with a few geometric parameters.

The Class A rules include the concept of a design life - the amount of time a component is allowed to remain in service. The rules deal with high temperature components where the actual component service life is always limited by creep rupture and the degradation of material properties with time - no high temperature component can be expected to last indefinitely. A reasonable metric for the Section III, Division 5, Subsection HB, Subpart B design margin is the ratio between the actual, expected component life and the design life. This is the metric applied in this report.

A margin assessment could be completed in two ways: deterministic or probabilistic. A deterministic analysis compares the best-estimate for the actual life of the component 
against its design life. A probabilistic assessment would consider how the variability in material properties, component geometry, and loading affects the expected operating life. This type of analysis could lead to the probability distribution function, $\rho(t)$, giving the marginal probability of the component failing in $t$ years after construction. The cumulative probability

$$
P=\int_{0}^{t_{d e s i g n}} \rho(t) d t
$$

where $t_{\text {design }}$ is the design life, gives the probability of premature failure, which would be the corresponding probabilistic metric of design margin.

For components where the uncertainty in the actual service life is low there would be little difference between a deterministic and a probabilistic assessment of margin. This report provides a deterministic method for assessing the design margin in the ASME rules. However, developing efficient methods for a probabilistic margin assessment should be a long term research objective, as this type of assessment gives more complete information compared to a deterministic assessment. Chapter 4 describes the challenges that would need to be overcome in developing a comprehensive probabilistic margin assessment method.

The deterministic assessment developed here needs a tool for predicting the actual, expected (mean-property) life of a component. This report uses continuum damage mechanics and a full thermal-mechanical finite element analysis of the component to provide this tool. Simple methods of this type are often applied in high temperature design (e.g. 1,2). In fact, the ASME rules provide a method for design by inelastic analysis, which includes a very simple damage calculation. The difference is that the continuum damage model developed here is comprehensive - it covers all the failure modes included in the ASME rules and covers a reasonably-complete range of component operating conditions. Moreover, it integrates into material deformation model, so that, for example, creep deformation becomes easier as the strength of the material decreases. The key to the approach is a work-based damage model that captures the available tensile, creep, fatigue, and creep-fatigue data. This type of damage model has been applied to high-temperature components in the past [3, 4], but this report is the first to use the method to predict the margin of the ASME rules for a particular component.

The report focuses on Alloy 617, both because it is a likely structural material for one candidate advanced high temperature reactor design - the Very High Temperature Reactor (VHTR) - and because an extensive, complete experimental database is available from the recent Alloy 617 Code qualification effort. The method for margin assessment developed here is comprehensive - it can be used to assess the margin for all of the different design limits covered by the Division 5 rules - but the focus of the report is on the creep-fatigue rules. The report focuses on creep-fatigue because it is the design limit most unique to the Class A design approach and because it tends to be the most limiting and most difficult design failure mode in a Division 5 analysis.

Chapter 2 describes the development, calibration and validation of the Alloy 617 damage model. Chapter 3 then applies the model to a margin assessment of the inner tube of a notional Alloy 617 hot gas duct in a VHTR. The assessment focuses on the creep-fatigue rules and projects an expected design margin of 10 on life - that is, the component is expected to have an actual life 10 times greater than the ASME design life. This order of 
magnitude in design margin arises from conservative assumptions used in constructing an approximation of creep and plastic material behavior starting from an elastic analysis and, especially, the conservative factors applied to the analysis results when calculating creep (stress increased by a factor of 1.11) and fatigue damage (strain range increased by a factor of 2, cycles to failure reduced by a factor of 20). Finally, Chapter 4 summarizes the report and discusses future work focused on extending the current, deterministic method to cover additional materials and component types and identifies the challenges in a developing a probabilistic margin assessment method for high temperature structural components. 



\section{A damage model for Alloy 617}

A complete damage model for a metallic material in high temperature service would include degradation caused by both cyclic and steady loading conditions. Creep-fatigue experiments cover both types of loading and so the focus of this chapter is on developing a damage model for Alloy 617 that can represent the available experimental creep-fatigue data. A key aspect of selecting a model for assessing the margin of the ASME design methods is finding a model form that can reasonably extrapolate to component loading conditions. Components typically experiences lower stresses and strain ranges and longer hold times when compared to creep-fatigue tests. However, as detailed below, the model developed here for Alloy 617 also represents material failure under creep conditions, as well as failure at much faster strain rates during standard tension tests. As such, the model here comes close to a "universal" high temperature damage model, which could be used to assess the margin in the ASME rules covering steady-state conditions, in addition to the creep-fatigue margin assessment completed in the current report.

The complete model developed here has two components: a damage model representing material failure and a base, underlying inelastic deformation model representing strain accumulation and stress redistribution in Alloy 617. The subsequent discussion covers the development validation of the damage model. The deformation model was previously developed for Alloy 617 by Walker and Sham at Oak Ridge National Laboratory [5]. A companion report discusses the implementation and validation of the deformation model [6].

\subsection{Damage model derivation}

The basic concept of the model is that at a constant work rate the material will fail when it dissipates a critical amount of inelastic energy (or, equivalently, does a critical amount of work), i.e. when

$$
W=\int \dot{W} d t=\int \boldsymbol{\sigma}: \dot{\varepsilon}_{\text {inelastic }} d t=W_{\text {crit }}
$$

where $W$ is the work, $\dot{W}$ is the work rate, $\boldsymbol{\sigma}$ is the stress tensor, $\dot{\boldsymbol{\varepsilon}}_{\text {inelastic }}$ is the inelastic, nonrecoverable, strain rate, $W_{\text {crit }}$ is the critical work to failure, and : represents double tensor contraction. However, when the material deforms at different work rates during loading the critical work varies with the work rate. We could posit a damage model where the $D=0$ means the material is completely undamaged and $D=1$ means the material has zero load carrying capability. Applying this idea to the work-based damage model gives the equation:

$$
\begin{gathered}
D=\int \dot{D} d t \\
\dot{D}=\frac{\dot{W}}{W_{\text {crit }}(\dot{W})}
\end{gathered}
$$

where $W_{\text {crit }}(\dot{W})$ is the work to failure given as a function of the current inelastic work rate. Conceptually, calibrating this type of model is simple: given a collection of experiments, defined as time, strain, stress histories which end in the failure of the material and occur 
at a constant inelastic work rate, calculate the total work dissipated over each test and plot this critical work to failure as a function of the work rate. Unfortunately, no standard experimental test deforms the material at a constant work rate. Instead, a more complicated process is needed to calculate the model, described in Section 2.2.

One further modification improves the detailed model predictions relative to experiments. The basic model described in Eq. 2.3, when implemented in the context of standard continuum damage mechanics, would degrade the material's load carrying capability linearly as work accumulates in the material. In experiments typically degradation in the material strength occur slowly at first and then more rapidly near the end of the test. A simple modification to Eq. 2.3 represents this behavior:

$$
\dot{D}=n D^{\frac{n-1}{n}} \frac{\dot{W}}{W_{\text {crit }}(\dot{W})}
$$

where $n$ is a parameter. For a hypothetical experiment deforming the material at a constant work rate to failure the accumulated work at failure can be calculated by solving the ordinary differential equation:

$$
\begin{array}{rr}
\dot{D} & =\quad n D^{\frac{n-1}{n}} \frac{\dot{W}}{W_{\text {crit }}} \\
D(0) & = \\
0 .
\end{array}
$$

The solution to this equation is

$$
D(t)=\left(\frac{\dot{W}}{W_{\text {crit }}} t\right)^{n}=\left(\frac{W}{W_{\text {crit }}}\right)^{n}
$$

where $W$ is the accumulated work. At failure this equation gives

$$
1=\left(\frac{W_{\text {fail }}}{W_{\text {crit }}}\right)^{n}
$$

where $W_{\text {fail }}$ is the accumulated work at failure when $D=1$. This equation can be rearranged to

$$
W_{\text {fail }}=W_{\text {crit }}
$$

Therefore this variant of the model retains the idea of a critical work to failure. Figure 2.1 illustrates the advantage of this model. This figure shows a series of simulated tension tests extending to the failure of the material. All tests have the same inelastic deformation model and the same critical work to failure $W_{\text {crit }}$. The only thing that changes is the exponent $n$. As this parameter increases more and more of the effects of the accumulated damage are deferred towards the end of the material life. By tuning the parameter $n$ to experimental data the effects of damage can be aligned with the experiments. 


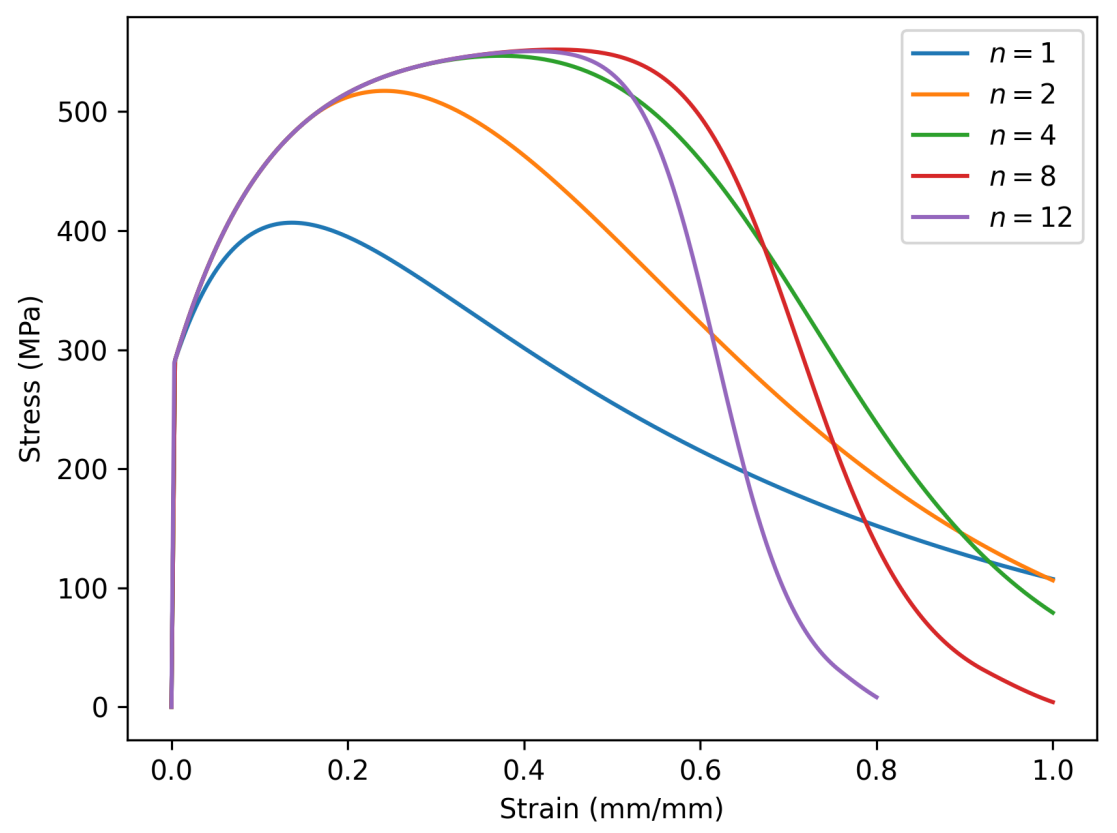

Figure 2.1: Example simulation demonstrating the effect of increasing the parameter $n$.

\subsection{Calibrating the damage model}

Two types of data were used to calibrate the model. The first were complete records of standard tension, creep, fatigue, and creep-fatigue tests conducted at INL. This dataset includes the full time, temperature, strain, and stress history of the experiment. This complete history can be used to calculate the instantaneous work rate as a function of time:

$$
\dot{W}=\sigma \dot{\varepsilon}_{\text {inelastic }}=\sigma\left(\dot{\varepsilon}-\dot{\varepsilon}_{\text {elastic }}\right)=\sigma\left(\dot{\varepsilon}-\frac{\dot{\sigma}}{E}\right) .
$$

The scalar form presented here is sufficient as all the test conditions are for uniaxial stress. In this expression $E$ is the material Young's modulus, which was taken from the Alloy 617 Code Case [7] as a function of temperature. The strain and stress rate can be calculated from the experimental data by finite differencing. The total work to failure is the integral of the work rate over the entire test time:

$$
W_{\text {fail }}=\int_{0}^{t_{\text {fail }}} \dot{W} d t .
$$

For experimental tests with a constant work rate we could associate $W_{\text {crit }}=W_{\text {fail }}$ and $\dot{W}_{\text {test }}=\dot{W}$. A regression analysis of plots showing the critical work versus the experiment work rate could provide the model for $W_{\text {fail }}(\dot{W})$.

However, none of the INL tests have a constant work rate. Instead, the experimental average work rate

$$
\dot{W}_{\text {test }}=\int_{0}^{t_{\text {fail }}} \dot{W} d t / \int_{0}^{t_{\text {fail }}} d t=W_{\text {fail }} / t_{\text {fail }}
$$




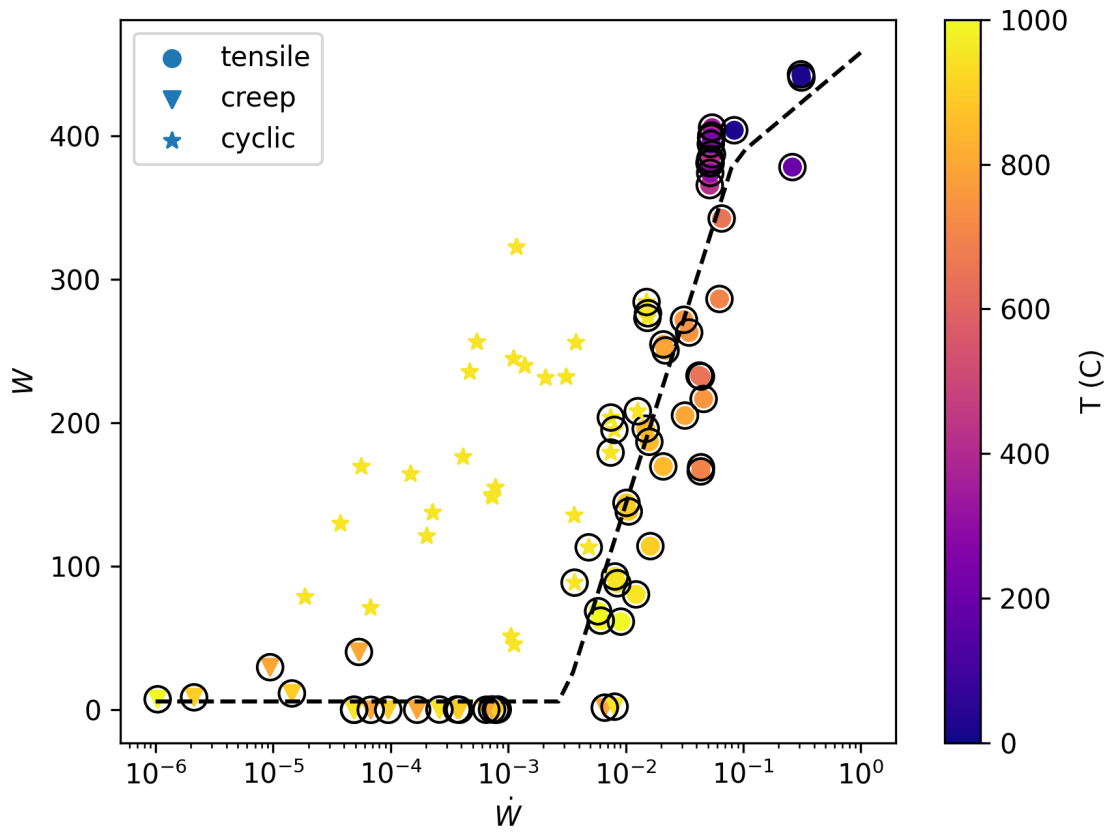

Figure 2.2: Initial damage model calibration to the full INL experimental data set. The figure shows points for the work-to-failure and average experimental work rate for all the tests. The tests corresponding to the circled symbols were used to fit the semilog trilinear initial correlation for $W_{\text {crit }}(\dot{W})$. The omitted tests are the cyclic experiments with holds (i.e. creep-fatigue tests). The data shows a moderate temperature effect, which is neglected for the current work.

versus the work to failure sets the model for $W_{\text {fail }}(\dot{W})$. This process excluded the creepfatigue testss. These tests deform the material at two very different work rates - very quickly during the loading period and very slowly during the holds - and so it is not reasonable to apply an average work rate for this type of test. Figure 2.2 plots the work to failure versus work rate relation derived from this process considering all the available INL 617 data. The plot includes points for the creep-fatigue tests using the experimental average work rate demonstrating that they do not overlap the rest of the data when plotted with the average of the slow and fast work rates. The data on this figure produces a model for $W_{\text {crit }}(\dot{W})$. The data suggests a sigmoidal response between a lower-shelf ductility at low work rates and an upper-shelf ductility at faster work rates. This type of response is typical for ductility-based methods [8]. The actual model approximates this sigmoid function with a trilinear semi-log response, plotted over the experimental data. The lower shelf branch is constant while the upper shelf branch has a positive slope.

The response of the failure model to long-term creep deformation is very sensitive to the slope and intercept of the lower-shelf branch. Creep tests occur at fixed stress. For the typical power law creep response

$$
\dot{\varepsilon}_{\text {inelastic }}=A \sigma^{n}
$$


for some prefactor $A$ and rate sensitivity $n$. The work rate is then

$$
\dot{W}=\sigma \dot{\varepsilon}_{\text {inelastic }}=A \sigma^{n+1} .
$$

The critical work to failure can be integrated as

$$
W_{\text {fail }}=\int_{0}^{t_{\text {fail }}} \dot{W} d t=A \sigma^{n+1} t_{\text {fail }}
$$

or

$$
t_{\text {fail }}=\frac{W_{\text {fail }}(\dot{W})}{A \sigma^{n+1}} .
$$

The challenge here is that as $\dot{W} \rightarrow 0$ the time to failure should increase to infinity, as the material is not deforming. However, even for a very small work rate $t_{\text {fail }}$ should be finite and could in fact be relatively small. The model must be able to discern between these two cases. Moreover, there are a only a few datapoints in this region of the critical work diagram, making it difficult to accurately constrain the failure model.

The Alloy 617 Code Case [7] collated a wide database of creep-rupture data for Alloy 617 - much wider than the very limited number of creep tests conducted at INL. However, unlike the INL creep deformations tests, full strain/time data for these experiments is not available. However, the Code Case did summarize the rupture data using the Larson-Miller [9] correlation:

$$
\log _{10} t_{R}=\left[\frac{a_{0}+a_{1} \log _{10} \sigma}{T}\right]-C
$$

with constants $a_{0}=32976.411, a_{1}=-5908.1030$, and $C=16.73049$, and with the temperature $T$ in kelvin. Ideally we could calibrate the lower-shelf branch of the failure model to this broader dataset.

Considering an idealized power law creep material, the critical work to failure should be

$$
\begin{gathered}
10^{-C} 10^{\frac{a_{0}+a_{1} \log _{10} \sigma}{T}}=\frac{W_{\text {fail }}}{\dot{W}} \\
W_{\text {fail }}=10^{-C} 10^{a_{0} / T}(\dot{W} / A)^{\frac{a_{1} / T}{n+1}} \dot{W} .
\end{gathered}
$$

Lumping the prefactors, this gives the model:

$$
W_{f a i l}=K \dot{W}^{\frac{a_{1} / T}{n+1}+1}
$$

which is a power law.

In actuality, Alloy 617 does not follow a power law creep relation. Instead it shows the typical pattern of a decreasing primary creep rate followed by an increasing tertiary creep rate. To match these conditions, we fit a power law through simulations of each creep condition, using the Walker inelastic model coupled to the work-based damage model to simulate the failure of the material. The power law parameters are those that best minimize the difference between the simulated rupture time and the Larson-Miller correlation for 20 creep simulations selected as follows: 


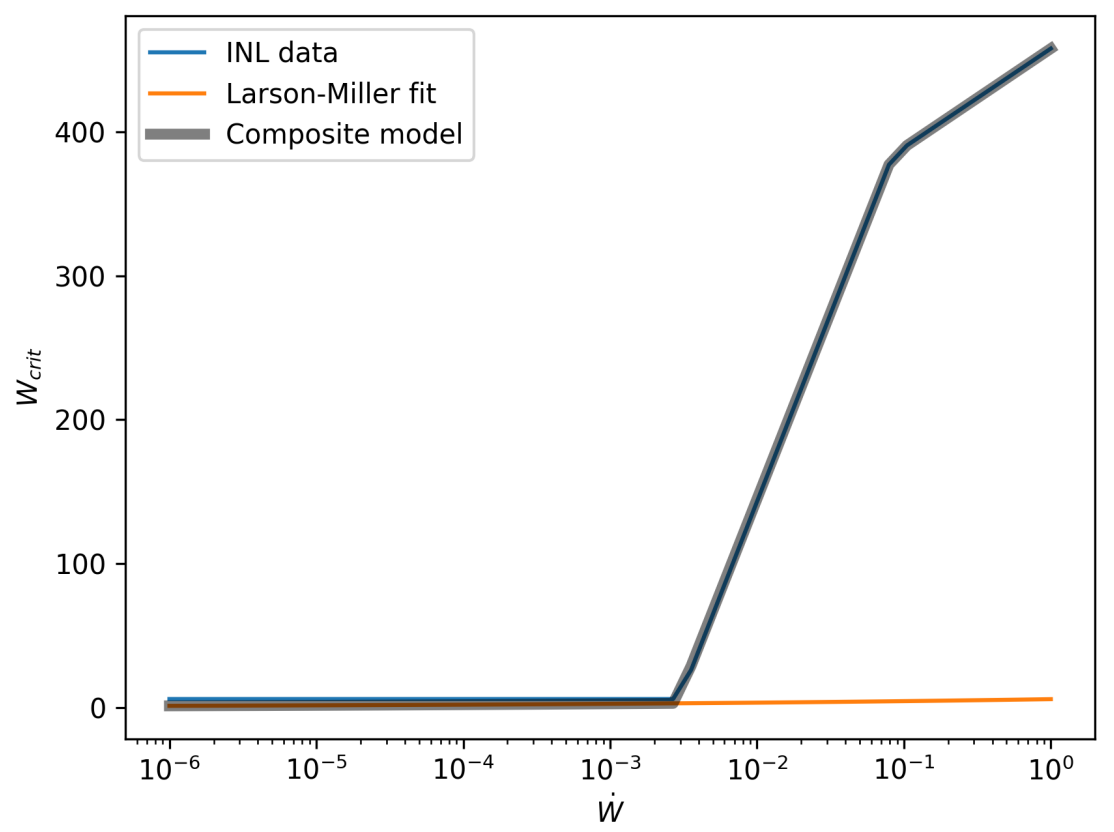

Figure 2.3: Composite model combining the fit to the full INL experimental data and the power law model for the lower-shelf ductility fit to the Larson-Miller correlation.

1. Select a target temperature of $950^{\circ} \mathrm{C}$, which is the temperature of most of the full INL creep-fatigue and fatigue experiments.

2. Using the Larson-Miller correlation (Eq. 2.17), generate 25 rupture stresses for equally log-spaced rupture times spanning from 10 to 300,000 hours.

3. Find the power law parameters $A$ and $n$ for critical work relation $W_{\text {crit }}=A \dot{W}^{n}$ that minimizes the squared difference between those 25 rupture times and the rupture time simulated with the Walker model coupled to the work-based damage model.

The final power-law parameters from this process are $A=5.768775$ and $n=0.119064$.

Figure 2.3 then combines the model generated using the full INL experimental data and the model generated by simulating creep tests and comparing to the A617 Larson-Miller correlation. While the difference between the power law fit from the Larson-Miller correlation and the constant value fit from the full creep tests seems small, capturing this small difference is necessary to accurately represent the creep test data. The inelastic damage model used in the margin assessment described in Chapter 3 uses the composite model combining the two sources of data, shown on Fig. 2.3.

The only remaining damage model parameter is the acceleration exponent $n$. This work uses $n=2$ based on a comparison between the model and experimental tensile flow curves and cyclic creep-fatigue test data at $950^{\circ} \mathrm{C}$. 


\subsection{Complete inelastic damage model}

The complete inelastic model combines the damage developed in the previous sections with the Walker inelastic deformation model for Alloy 617. A companion report [6] summarizes the derivation of the Walker model and validates it against an experimental database of high temperature Alloy 617 tests. This report therefore omits the details of the base inelastic deformation model.

The damage model modifies the constitutive response provided by the base model in the standard manner for continuum damage mechanics:

$$
\dot{\sigma}=(1-D) \dot{\sigma}_{\text {base }}
$$

where $D$ is the damage variable, defined previously, and $\dot{\sigma}_{\text {base }}$ is the viscoplastic response of the base deformation model, here the Walker model.

\subsection{Evaluating the damage model}

The inelastic damage model calibrated here is most accurate at $950^{\circ} \mathrm{C}$, as that is the temperature for most of the INL creep-fatigue test data. Figure 2.4 compares the predicted tensile curve using the complete inelastic damage model to the available INL experimental flow curves. Figure 2.5 makes a similar comparison for the available creep deformation data at $950^{\circ} \mathrm{C}$. The model accurately predicts the experimental flow curves, though the underlying Walker model slightly underpredicts the initial flow stress. The creep curve comparisons demonstrate that the model accurately recovers the Alloy 617 rupture database. The comparison to the available full creep curves is somewhat less accurate, though note that these creep tests did not keep a record of the material deformation all the way to failure.

These comparisons are not truly model validation as the tension test and creep test data was used to fit the critical work function. Figure 2.6 compares the actual experimental sample lives to the lives predicted by the model, including the INL creep-fatigue data not included in the model calibration data. The comparison shows a histogram of the value:

$$
\text { error }=D_{\text {exp }}-1
$$

where

$$
D_{\text {exp }}=\int_{0}^{t_{\text {exp }}} \frac{\dot{W}_{\text {exp }}}{W_{\text {crit }}\left(\dot{W}_{\text {exp }}\right)} d t
$$

where $\dot{W}_{\text {exp }}$ are the instantaneous experimental work rates, $t_{\text {exp }}$ is the experimental time to failure, and $W_{\text {crit }}$ is the critical work function detailed previously. Essentially this comparison calculates the predicted damage at the end of each experiment. If the damage model was perfect this value would equal 1.0 for all the tests. The error plotted on Figure 2.6 is then the error between the actual and predicted damage. This error is also the approximate relative error on cycles to failure for these tests.

Most of the experimental error is distributed about 0 (i.e. perfect accuracy) with a bias towards positive errors (i.e. the model over-predicts damage accumulation in the material). However, errors with values less than 1.0 on this plot suggest a relative error in the specimen 


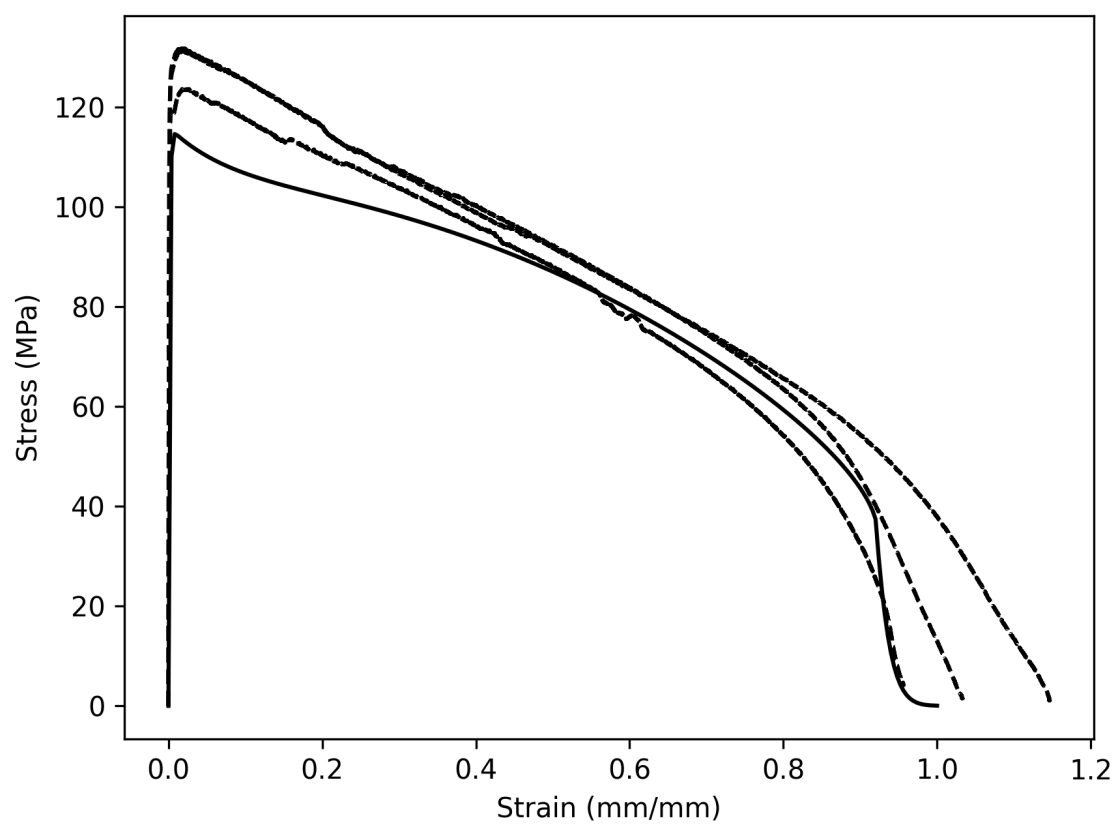

Figure 2.4: Comparison between the inelastic damage model and the available INL tensile curves at $950^{\circ} \mathrm{C}$. Model is the solid black line, experiments are the dashed lines.

life of less than a factor of 2 . This is not a significant error, as the batch-to-batch variation in the experimental life often exceeds a factor of 2 for creep or creep-fatigue tests. In addition, there is a limited number of experiments which are outliers with the model over-predicting damage accumulation by a factor of 4 . Reducing these outliers will be a focus of future work. 


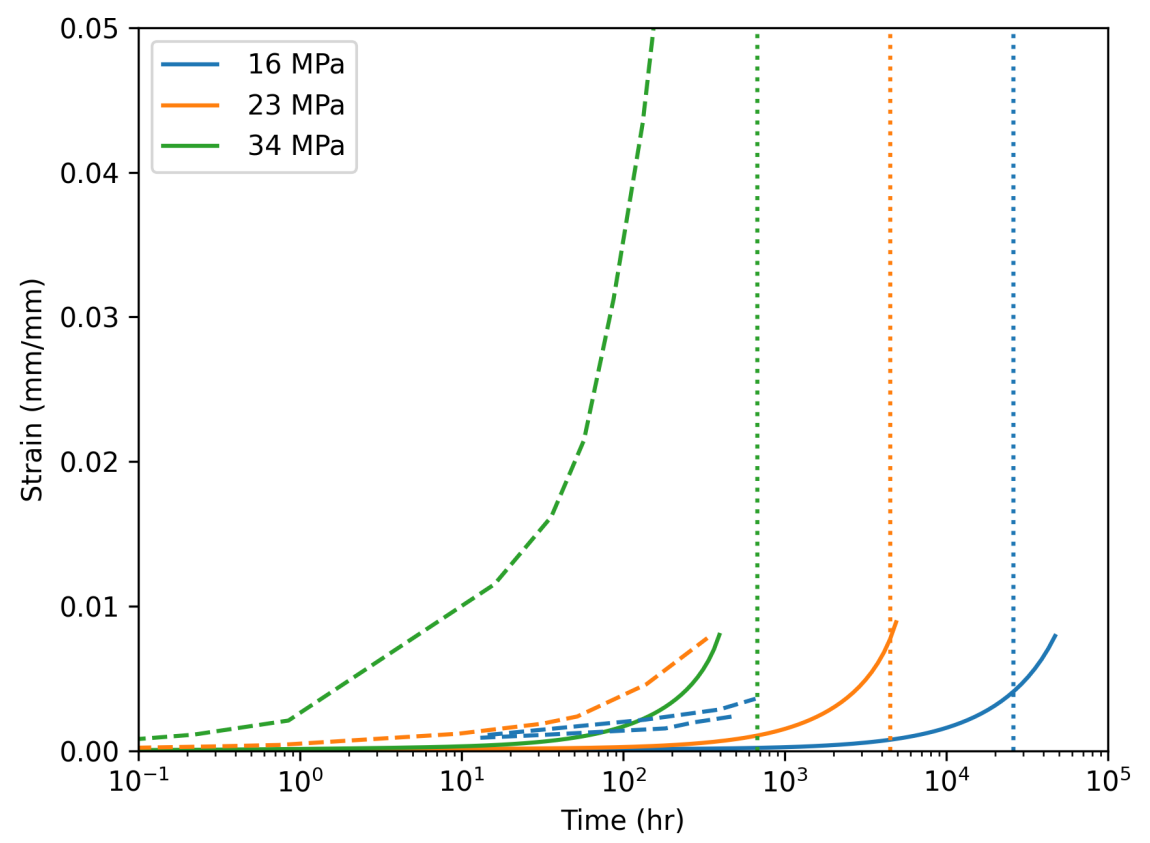

Figure 2.5: Comparison between the available creep curves and the model predictions at $950^{\circ} \mathrm{C}$. The solid lines give the model predictions for the corresponding stress levels. The dashed curves are the experimental creep curves. The dotted vertical lines show the rupture life predicted by the Larson-Miller correlation. 
An Initial Assessment of the Design Margins of Different ASME Section III, Division 5 Design Rules

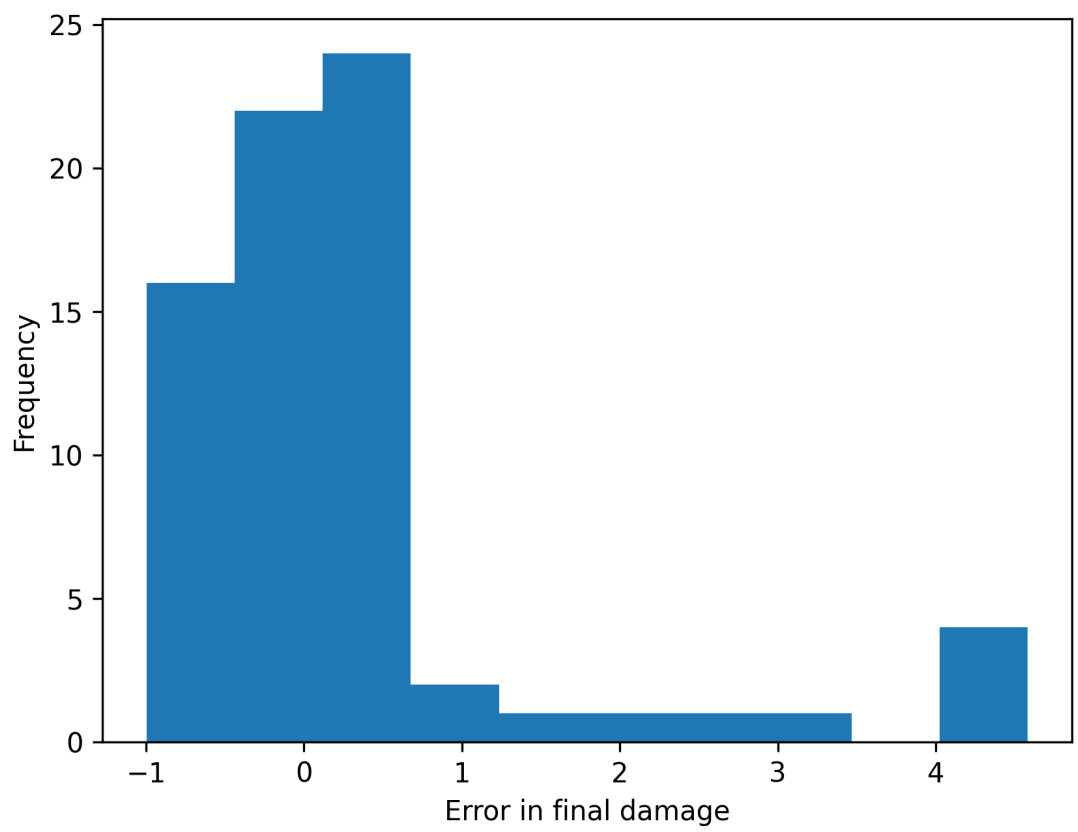

Figure 2.6: Comparison between the predicted and actual accumulated damage for all the experimental data, including the creep-fatigue tests. 


\section{Example margin determination}

\subsection{The sample problem}

The sample problem is based on a conceptual design of an Alloy 617 hot gas duct in a VHTR [10]. This component connects the reactor outlet to the intermediate heat exchanger (IHX). Its task is to carry very hot helium gas $\left(950^{\circ} \mathrm{C}\right)$ between the reactor and the heat exchange system. Because of the very high operating temperatures (metal temperatures reaching $850^{\circ} \mathrm{C}$ ) and pressure $(4 \mathrm{MPa})$ this component will need to be constructed from a material with excellent high temperature structural resistance, like Alloy 617 [11]. Figure 3.1 shows the geometry, both the full cross-section of a nominal co-axial double tube duct and the simplified structural model of the inner tube. The structural model approximates an emergency shutdown following a loss-of-insulation on the inner tube. The transient represented in Figure 3.1 would be a severe accident condition. In a full design this severe transient would be a relatively isolated event, interspersed with long periods of steady-state normal plant operation, and less severe normal shutdown transients. The overall life of the duct might be several hundred thousand hours. This analysis focuses only on this severe transients and looks at the creep-fatigue life of the system using the current Section III, Division 5, Subsection HB, Subpart B design rules and using the full inelastic damage model.

Table 3.1 lists the key geometric, loading, and thermal properties used in the analysis. The analysis neglects the small stress generated by the offsetting $4 \mathrm{MPa}$ pressures, focusing on the thermal stresses. A transient heat transfer simulation of the inner tube establishes the metal temperatures as a function of time. These metal temperatures were used in the ASME design and best-estimate damage analysis.

Figure 3.2 plots the temperature in the inner tube as a function of time at three locations (tube inner diameter, tube outer diameter, and halfway between). The temperature rapidly increases when the tube surface becomes exposed to the $950^{\circ} \mathrm{C}$ helium, and then decreases as the plant enters a safe shutdown mode. The transient parameters (Table 3.1) were selected to make the transient periodic, starting and ending at the same thermal conditions, to simplify the design and damage analysis.

In the subsequent structural analysis, the tube is treated as an axisymmetric slice of a long, straight section of piping. Generalized plane strain conditions were applied to the ends of the tube section in the analysis to represent the constraint of this structural configuration. The discretization divides the tube radially into 25 finite elements.

\begin{tabular}{lll}
\hline Parameter & Description & Value \\
\hline$t_{\text {tran }}$ & Time of the transient & 5 hours \\
$t_{\text {inner }}$ & Inner tube thickness & $20 \mathrm{~mm}$ \\
$h_{H e}$ & Convective heat transfer coef. He/A617 & $1500 \mathrm{~W} /\left(\mathrm{m}^{2} \mathrm{~K}\right)$ (approx. $\left.[12]\right)$ \\
$\alpha_{A 617}$ & Coefficient of thermal expansion for Alloy 617 & Temperature-dependent, see [7] \\
$k_{A 617}$ & Thermal conductivity for Alloy 617 & Temperature-dependent, see [7] \\
$d_{A 617}$ & Thermal diffusivity for Alloy 617 & Temperature-dependent, see [7] \\
\hline
\end{tabular}

Table 3.1: Key parameters for the sample problem. 


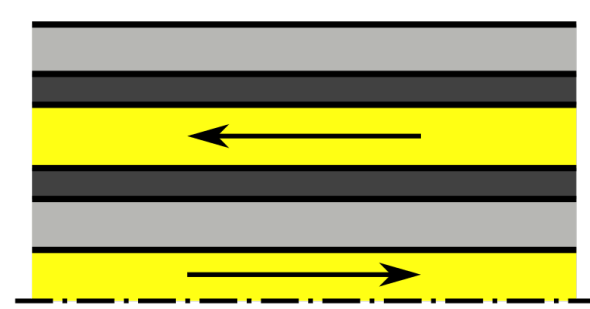

Full cross section

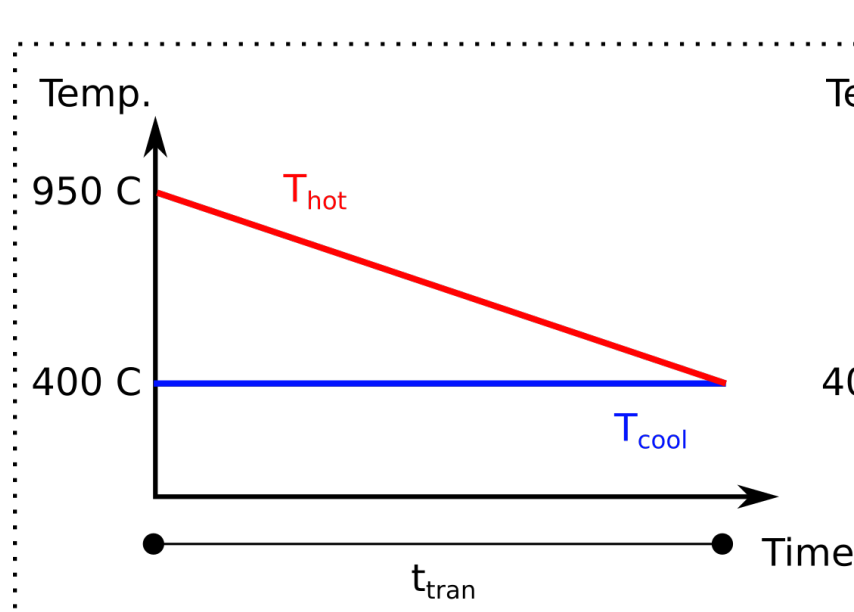

Time-dependent He temperatures Pressure tube Cool helium Inner tube Hot helium

Thermal insulation Thermal insulation

Figure 3.1: Sample problem used for the margin assessment. Figure shows a notional crosssection of a coaxial double tube duct along with the simplified structural model used in the margin assessment. 


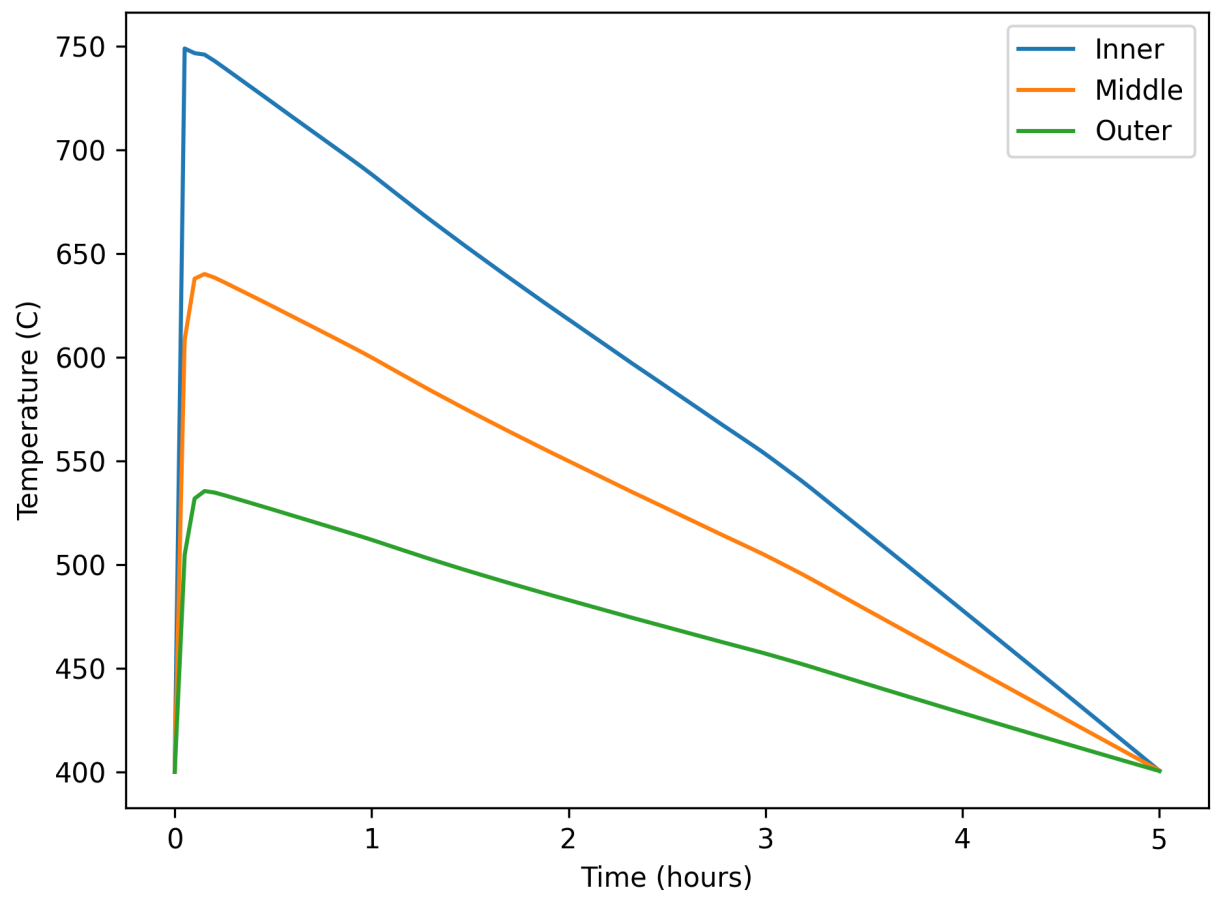

Figure 3.2: Temperatures at three locations across the inner tube, plotted as a function of time during the transient.

\subsection{ASME design by elastic analysis}

This section applies the ASME Section III, Division 5, Appendix HBB-T [13] design-byelastic analysis rules to predict the maximum allowable cyclic life of the sample problem described in the previous section. As the material here is Alloy 617, this analysis refers to the Alloy 617 Code Case [7] for the required material properties.

The ASME rules require a linear elastic stress analysis of the component. This analysis requires temperature-dependent values of the Alloy 617 Young's modulus and Poisson's ratio, again found in the Alloy 617 Code Case. Figure 3.3 shows the elastically calculated hoop stress in the inner tube as a function of time during the transient at three locations (tube inner diameter, tube outer diameter, and halfway in between). The complete analysis was 3D and so includes the radial and axial stresses, in addition to the hoop stresses shown on the figure. The design by elastic analysis rules require classifying this stress into primary, secondary, and peak components. In this particular example this is trivial as the primary stress is zero (neglecting the small stresses generated by equal pressures operating on either side of a thick-walled vessel) and the peak stress is zero because there are no stress concentrations in the problem. All the stresses are then secondary.

The Section III, Division 5, Subsection HB, Subpart B design rules are too complicated to briefly summarize here. However, there are a few key points for this particular design analysis:

- This example only considers the ASME creep-fatigue design rules. The full Section 


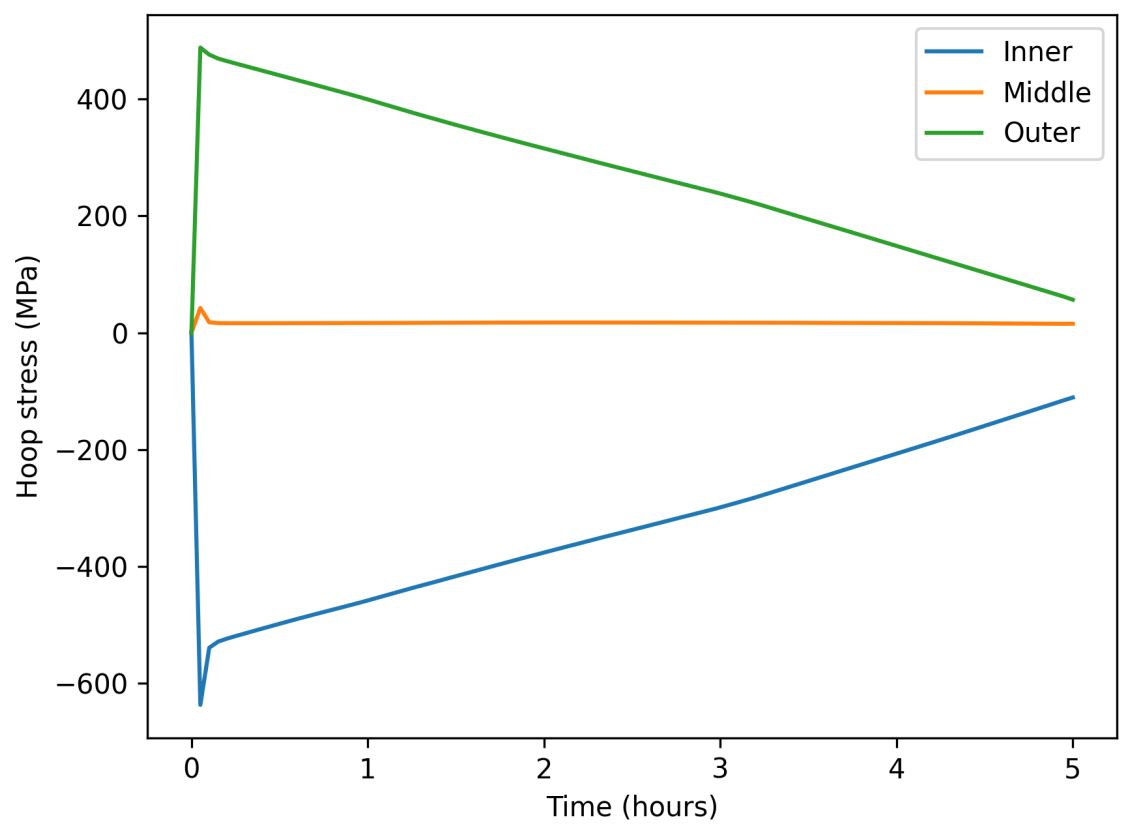

Figure 3.3: Elastically-calculated stresses in the inner tube.

III, Division 5, Subsection HB, Subpart B design process requires considering several design conditions:

- Primary load design

- Buckling

- Strain accumulation

- Creep-fatigue

- Of these four design conditions, only strain accumulation and creep-fatigue are relevant to the inner tube design, as the pressure-induced stresses are essentially zero for this transient.

- The analysis only considers the number of repetitions of this particular, severe transient allowed by the ASME rules. A full design analysis would need to consider all potential upset conditions as well as the loading induced by standard operating conditions.

- The actual design analysis calculates the creep and fatigue damage accumulated in a single repetition of the loading cycle and then extrapolates these values to the maximum number of transient repetitions allowed by the ASME rules. This approach is equivalent to a full analysis for this case, as the design analysis is linear elastic, the thermal conditions are periodic, and the analysis results can be superimposed.

A computer tool produced at ANL as part of an NRC-funded project was used to execute the ASME analysis. The thermal analysis was completed with an in-house heat transfer 


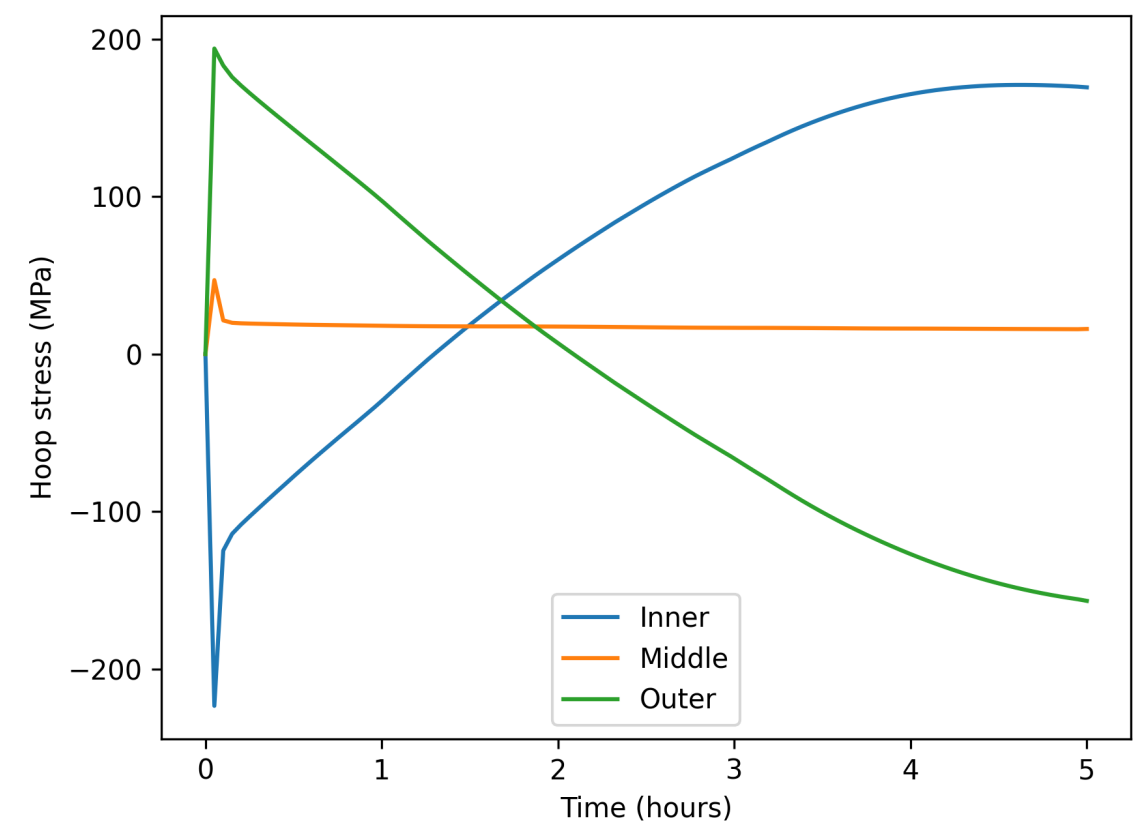

Figure 3.4: Stresses from the full damage calculation over the first repetition of the loading transient.

solver for cylindrical coordinates. The structural analysis used the NEML framework and material models (https://github.com/Argonne-National-Laboratory/neml).

The results of this design calculation were that the inner tube can withstand 5 repetitions of the loading transient according to the ASME rules.

\subsection{Inelastic damage modeling}

Compared to the ASME design by elastic analysis rules a best-estimate of the creep-fatigue life of the component is straightforward: simulate multiple repetitions of the transient loading until the damage in the component reaches 1.0 at any location through the tube thickness. In practice, the cost of this numerical simulation can be reduced by monitoring the increase in the damage variable at each location in the component as a function of cycle count and then extrapolating to the critical value of damage once the damage accumulation becomes steady as a function of cycle count. This simulation again used the NEML framework and the in-house heat transfer code. The analysis uses the full inelastic damage model developed in Chapter 2.

Figure 3.4 plots the hoop stress during the first cycle for this full inelastic simulation. The initial values are lower than in the linear elastic simulation (Fig. 3.3) because the inelastic model yields. Later during the transient the stress distribution is significantly different then the elastic stress distribution, as creep causes stress redistribution across the inner tube wall.

Figure 3.5 then shows the extrapolated damage at failure through the tube thickness. The model predicts that damage will concentrate on the tube inner (hot) wall, likely manifesting 


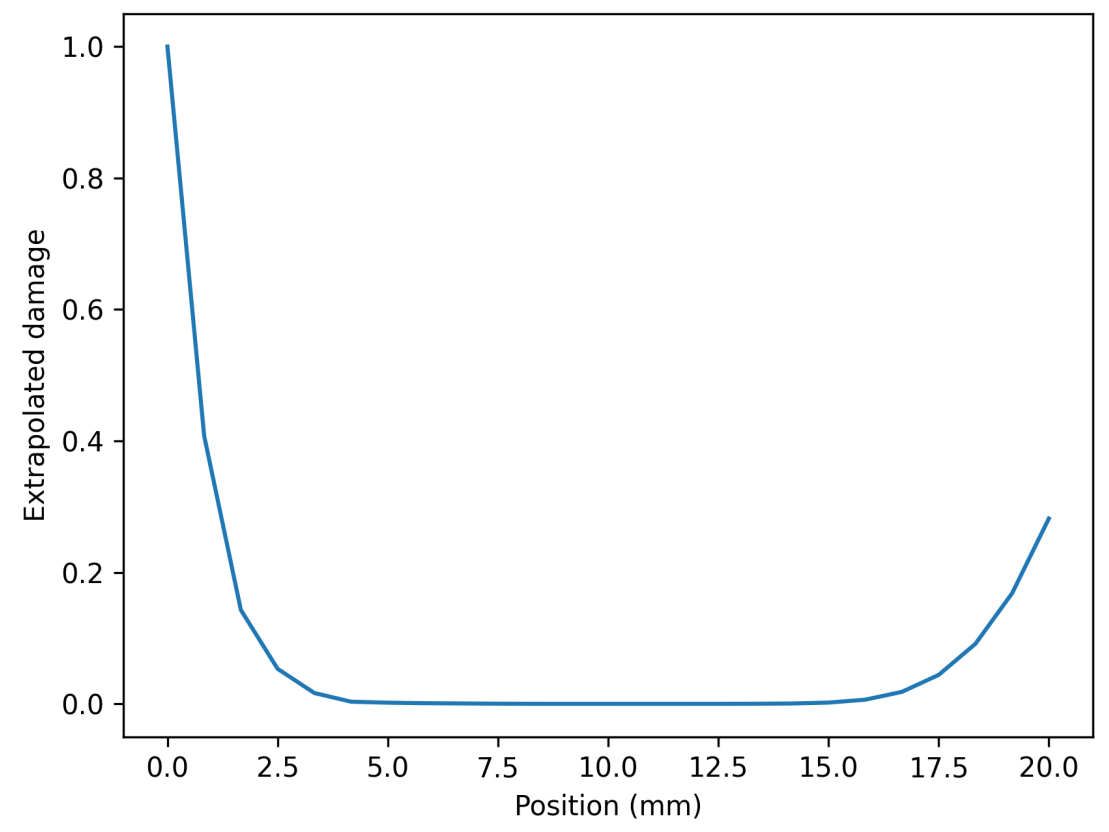

Figure 3.5: Extrapolated damage at failure plotted through the tube thickness.

as a creep-fatigue crack initiating from this surface.

The full damage predicts the component could withstand 51 repetitions of the transient.

\subsection{Margin assessment}

The margin in the ASME design by elastic analysis for this particular example is a factor of $51 / 5=10.2$. This suggests that for Alloy 617 at these conditions the margin in the ASME creep-fatigue rules is roughly a factor for 10 on life - the component will last ten times longer than the ASME design life.

The analysis method developed here could form the basis of a more comprehensive evaluation of the ASME design margin. The inelastic damage model described in this report is suitable for assessing the margin in all the ASME design conditions, not just the creep-fatigue rules. For example, a steady-state analysis of the component could be used to assess the ASME primary load design methods and the damage model accurately predicts steady-state creep rupture.

A comprehensive margin analysis would need to consider all the ASME Class A materials, cover a wide range of temperatures and loading conditions, and consider a wide variety of component types. Given that even a single full inelastic damage analysis of a single component requires a substantial amount of analyst effort, such a comprehensive analysis would require a substantial program of work. A more focused analysis could consider a narrower range of components and materials, for example focusing on a particular reactor type. 
The main barrier to starting such a margin assessment program is developing an adequate full inelastic damage model for the material, covering the required temperature range. The Alloy 617 model developed here covers the available experimental data with reasonable accuracy. However, the INL experimental database is biased towards relatively high temperatures, compared to expected operating temperatures in VHTR designs, and to short test durations compared to reasonable component design lives. Moreover, the INL database containing full (time,strain,stress, temperature) hysteresis data is exceptional - it does not exist for any of the other Class A materials. Additionally, this example and the damage model considered here does not consider weldments. Experimental data with the detail required to develop a full damage model for weldments or even just weld material is not available. This is a substantial gap, as welds are often the weakest link in actual high temperature components.

Finally, a more complete margin assessment would quantify the ASME design margin using a statistical approach, as discussed in the introduction. This would account for uncertainty in the base material properties as well as uncertainty in the applied loads and the component geometry, through construction tolerances. Chapter 4 discusses potential future work on this topic.

Despite these caveats, the sample margin assessment method developed in this report is a valuable first step in developing a process for quantifying the margin in the design rules. Though this report considers only a single component and loading, selected in part to load the material in conditions comparable to the experimental data used to produce the damage model, the margin of 10 on design life may be more generally applicable to the ASME creep-fatigue design rules. This analysis suggests that the ASME design rules are adequately conservative and that room exists to further optimize the design rules to produce more efficient component designs. 



\section{Conclusions and future work}

This report describes a deterministic method for assessing the design margin of the Section III, Division 5, Subsection HB, Subpart B ASME Boiler and Pressure Vessel Code rules for the design of Class A high temperature reactor components. The method applies a complete inelastic damage model of the component to determine the expected time-to-failure and compares this value to the component design life. The focus here was on the creep-fatigue rules, but the method could be applied to any of the Class A design limits. The sample component evaluated here has a margin of 10 on design life, which is likely representative of the conservative ASME rules.

The immediate next step would be to apply this process to the other design limits and to other representative Alloy 617 components. The deterministic approach developed here could be extended to other Class A materials. One challenge is that the record of the mostly historical testing database for these materials is incomplete. Full records that could be used to calculate the dissipated work are unavailable for most of the tests.

Longer term, a probabilistic assessment of margin would provide a better description of the Subsection HB, Subpart B design margin. The probability of premature failure could be used to both assess the margin in the current rules and to tune the rules to better reflect the safety significance of individual components. However, there are a number of challenges in developing a probabilistic assessment method for high temperature components:

- The most straightforward extension of the deterministic model developed here would be an inelastic damage model with model parameter probability distributions that in turn describes the distribution of deformation and failure data in tests.

- Calibrating these types of models is extremely difficult. Current efforts at ANL to calibrate probabilistic models using Bayesian analysis have demonstrated success for simple creep models, but capturing uncertainty in cyclic plasticity and failure is much more challenging.

- Monte Carlo analysis could be used in conjunction with this type of model to determine the component marginal failure probability distribution. However, this Monte Carlo analysis would be extremely computationally expensive, as it would require running thousands of full inelastic simulations of the component through the entire component loading history.

- The uncertainties in component loading conditions and actual, as-constructed geometric tolerances are not well quantified.

- There are very few statistical descriptions of high temperature material properties, like the properties controlling the creep deformation and failure of materials. Standard statistical methods could be applied to this data but there has been little previous work done for the ASME Class A materials.

- Simplified methods, for example deterministic methods linked to component failure statistics like Load and Resistance Factor Design (LRFD) approaches, have not been developed for high temperature components. 
An Initial Assessment of the Design Margins of Different ASME Section III, Division 5 Design Rules September 2020

Future work should address these challenges and work towards a probabilistic method for assessing the margin in high temperature design rules, with the goal of developing deterministic design methods better tailored to the margin required for individual plant components and systems. 
An Initial Assessment of the Design Margins of Different ASME Section III, Division 5 Design Rules September 2020

\section{Acknowledgments}

The research was sponsored by the U.S. Department of Energy, under Contract No. DEAC0206CH11357 with Argonne National Laboratory, managed and operated by UChicago Argonne LLC. Programmatic direction was provided by the Office of Nuclear Reactor Deployment of the Office of Nuclear Energy. The authors gratefully acknowledge the support provided by Sue Lesica, Federal Manager, Advanced Materials, Advanced Reactor Technologies (ART) Program, Diana Li, Federal Program Manager, ART Gas-Cooled Reactors (GCR) Campaign, and Gerhard Strydom of Idaho National Laboratory, National Technical Director, ART GCR Campaign. 



\section{Bibliography}

[1] M. C. Messner and T.-L. Sham. Inelastic analysis procedure based on the Grade 91 unified viscoplastic constitutive model for ASME implementation. Technical report, Argonne National Laboratory, ANL-ART-167, Lemont, IL, 2019.

[2] M. C. Messner, V.-T. Phan, and T.-L. Sham. A Unified Inelastic Constitutive Model for the Average Engineering Response of Grade 91 Steel. In Proceedings of the 2018 ASME Pressure Vessels and Piping Conference. PVP2018-84104, 2018.

[3] R. P. Skelton. The energy density exhaustion method for assessing the creep-fatigue lives of specimens and components. Materials at High Temperatures, 30(3):183-201, 2013.

[4] G. Douzian, O. Muránsky, J.J. Kruzic, R.N. Wright, and W. Payten. Assessment of modelling methodologies for prediction of high-temperature creep-fatigue behaviour of Alloy 617. International Journal of Pressure Vessels and Piping, page 104150, 2020. ISSN 03080161. doi: 10.1016/j.ijpvp.2020.104150. URL https://doi.org/10.1016/j.ijpvp.2020.104150.

[5] T.-L. Sham and Kevin P. Walker. Preliminary Development of a Unified Viscoplastic Constitutive Model for Alloy 617 With Special Reference to Long Term Creep Behavior. In Fourth International Topical Meeting on High Temperature Reactor Technology, pages $81-89,2008$.

[6] M. C. Messner and T.-L. Sham. Initial High Temperature Inelastic Constitutive Model for Alloy 617. Technical report, Argonne National Laboratory, ANL-ART-195, Lemont, IL, 2020.

[7] American Society of Mechanical Engineers. Case N-898: Use of Alloy 617 (UNS N06617) for Class A Elevated Temperature Service Construction: Section III, Division 5, 2019.

[8] M W Spindler. The multiaxial creep ductility of austenitic stainless steels. Fatigue and Fracture of Engineering Materials and Structures, 27(4):273-281, 2004. ISSN 8756758X. doi: 10.1111/j.1460-2695.2004.00732.x.

[9] F. R. Larson and J. Miller. A time-temperature relationship for rupture and creep stresses. Transactions of the ASME, 74:765-771, 1952.

[10] Makoto Hishida, Kazuhiko Kunitomi, Ikuo Ioka, Koji Umenishi, Toshiyuki Tanaka, Hiroaki Shimomura, and Konomo Sanokawa. Thermal performance test of the hot gas ducts of HENDEL. Nuclear Engineering and Design, 83(1):91-103, 1984. ISSN 00295493. doi: 10.1016/0029-5493(84)90034-7.

[11] K. Natesan, A. Purohit, and S.W. Tam. Materials Behavior in HTGR Environments. Technical report, Argonne National Laboratory, NUREG/CR-6824, ANL-02/37, 2003.

[12] Qiusheng Liu, Li Wang, Akihiro Mitsuishi, and Makoto Shibahara. Transient heat transfer for helium gas flowing over a horizontal cylinder in a narrow channel. Experimental Heat Transfer, 30(4):341-354, 2017. 
[13] American Society of Mechanical Engineers. Section III, Division 5. In Boiler and Pressure Vessel Code. 2019. 



\section{Argonne}

\section{Applied Materials Division}

Argonne National Laboratory

9700 South Cass Avenue, Bldg. 212

Argonne, IL 60439

www.anl.gov 